\title{
Parametric Image Formation Using Clustering for Dynamic Cardiac SPECT
}

\author{
Harshali Bal, Edward V. R. DiBella, Member, IEEE and Grant T. Gullberg, Senior Member, IEEE
}

\begin{abstract}
Dynamic cardiac SPECT imaging can provide quantitative and possibly even absolute measures of physiological parameters. However, a dynamic cardiac SPECT study involves a number of steps to obtain estimates of physiological parameters of interest. One of the key steps involves the selection of regions of interest. In the past, this has been done manually or by using a semi-automatic method. We propose to use cluster analysis to segment the data to obtain improved parameter estimates. The algorithm consists of using a standard k-means clustering followed by a blood input finetuning procedure using fuzzy k-means performed to obtain a more accurate blood input function. Computer simulations were used to test the algorithm and to compute bias in kinetic rate parameters with and without the use of blood input fine-tuning. This was followed by performing eight studies in three canines and three studies in two patients with a dynamic cardiac perfusion SPECT protocol. The short-axis slice image data were used as input for the cluster analysis program as well as for a previously validated semi-automatic method. All of the time activity curves were fit to a two-compartment model. Parametric images of the wash-in rate parameter were obtained after cluster analysis. The wash-in rate estimates from the selected regions of interest with both of the methods were compared using microsphere derived flows as a gold standard in the case of canine studies. Our results suggest that in regions with low noise, cluster analysis provides parameter estimates comparable to the semi-automatic method in addition to providing improved visual defect localization and contrast. Moreover, the clustered curves have less noise and yield reasonable fits where with the semi-automatic method the fitting routine sometimes failed to converge. The use of clustering also required less manual intervention than the semi-automatic method. These results indicate that use of clustering may bring dynamic cardiac SPECT closer to clinical feasibility.
\end{abstract}

\section{INTRODUCTION}

$\mathrm{W}$ ORK done by several investigators has shown the potential of dynamic cardiac SPECT to provide quantitative measures of myocardial physiological parameters [1]-[4]. Our group has shown that the use of tracers such as

Manuscript received December 2, 2002. This work was supported by NIH under Grant R01 HL50663 and by U.S. Department of Energy contract DEAC03-76SF00098.

$\mathrm{H}$. Bal is with the Department of Bioengineering, University of Utah, UT 84112 USA (e-mail: khare@eng.utah.edu)

E. V. R. DiBella is with the Department of Radiology, University of Utah, UT 84108 USA (e-mail: ed@mirl.med.utah.edu)

G. T. Gullberg is with the Department of Nuclear Medicine and Functional Imaging, E. O. Lawrence Berkeley National Laboratory, Berkeley, CA (e-mail: gtgullberg@lbl.gov)
${ }^{99 \mathrm{~m}}$ Tc-labelled teboroxime (teboroxime) and Thallium-201 $\left({ }^{201} \mathrm{Tl}\right)$ with dynamic cardiac SPECT yields parameters that correlate with myocardial blood flow in addition to providing improved defect contrast [2]-[4].

The processing of dynamic data consists of reconstructing the projection data and selecting regions of interest to obtain time-activity curves that are then fit to appropriate models representing the tracer kinetics. In the case of some dynamic PET as well as SPECT studies, pixel-wise fitting of the timeactivity curves has been used [5], [6]. The presence of high statistical noise in dynamic cardiac SPECT however, has led to using regions of interest (ROIs) that are typically about $1.5-2.5 \mathrm{~cm}^{3}$. This selection of ROIs is done either manually or by using a semi-automatic method. The semi-automatic method selects myocardial regions from the short-axis slices based on a histogram specification technique [7], while the blood input function is obtained by manual region selection. This can result in high statistical noise in the resulting timeactivity curves due to non-optimal region selection causing occasional failure of the fitting routine to converge.

In this work, we develop a clustering technique to segment dynamic cardiac SPECT data and then form parametric images by fitting the resulting clusters to a two-compartment model. Different approaches to cluster analysis have been used previously by others with dynamic FDG PET studies to segment the data [8], [9].

Our main aim is to develop an automated method for segmenting dynamic cardiac SPECT data using clustering and also obtain parametric maps with improved estimates reflecting blood flow. The two tracers used for this investigation are teboroxime and ${ }^{201} \mathrm{Tl}$. The method is validated with the use of simulations and real studies employing canines and patients and the rate estimates are compared to that obtained with the present semi-automatic technique. Microsphere derived flows are used as a gold standard for comparison in the case of canine studies.

\section{METHODS}

\section{A. Algorithm}

The process of segmenting the time-activity curve data from dynamic SPECT studies was done using a clustering technique. First, a standard k-means procedure [11] was used to segment the time-activity curves based on minimizing the least-squares distance between the curves. The standard k- 
means clustering minimizes $\sum_{p} \sum_{n \in C_{p}}\left\|a_{n}-\mu_{p}\right\|^{2}$ where $a_{n}$ is the time-activity curve for spatial location $n$ and $\mu_{p}$ is the average curve of the members of the $p^{\text {th }}$ cluster $C_{p}$ [10]. The algorithm first selects an initial set of cluster centers based on maximizing the least-squares distance. The time-activity curve from each pixel, represented by the $a_{n}$ vector, is then compared to each cluster and assimilated with the cluster having minimum least-square distance. The cluster centroid is then recomputed as the mean of the curves constituting the given cluster. This process is repeated with the new updated cluster centroids till the time-activity curves stop changing clusters.

The second step consists of employing a fuzzy k-means procedure aimed at obtaining a more accurate blood input function. We refer to this as the blood input fine-tuning. In this method, the clusters obtained from the standard k-means process were used to obtain graded membership of each pixel with the cluster means expressed below: [11]

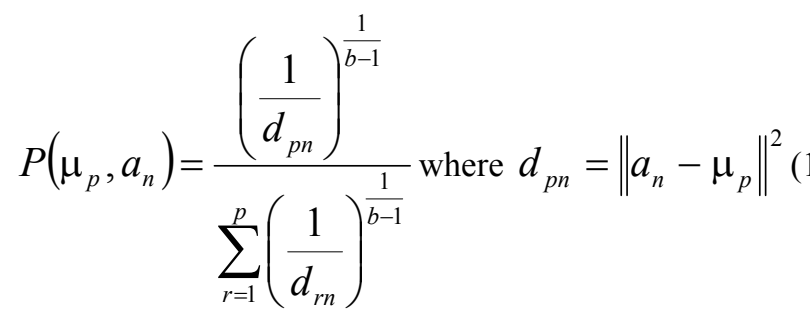

Here, $d$ is the distance measure similar to that used in the standard k-means case above and $b$ is the fuzzification parameter that has been set to 1.1. The value of $b$ is dependent on the noise level but changes in this parameter were not found to affect the results for our studies.

The use of the left ventricular blood cluster from the standard k-means clustering may result in an input function being contaminated by the surrounding myocardial tissue uptake, particularly when few clusters are used. The use of the fuzzy k-means gave a membership function for each pixel along with the cluster means that was in turn used to select the most likely blood pixels from the blood cluster. The selection of blood pixels was performed by using a probability threshold.

An example of the application of the algorithm consisting of the two steps as explained earlier with dynamic teboroxime canine data is shown in Fig. 1. Thirty clusters were used to segment the 4D short-axis data and the resulting clusters fit to a two-compartment model to obtain the kinetic rate parameters. The figure shows the summed static shortaxis images as well as the corresponding wash-in parametric image with clustering. Outliers consisting of blood regions were set to zero in the case of the wash-in parametric image.

\section{B. Simulations}

Computer simulations modeling the myocardial uptake of teboroxime were performed to test whether the blood input function from using only standard k-means clustering results in bias in the parameter estimates. The blood input function from a patient study with a bolus infusion was used as a reference to obtain a functional form of the blood input function. The blood input function used was:

$$
\left.\begin{array}{cc}
450 \cdot \frac{t}{20} & t \leq 20 s \\
b(t)=400 \cdot e^{-(0.3 \cdot(t-20))}+80 \cdot e^{-(0.03 \cdot(t-20))} & t>20 s \\
+60 \cdot e^{-(0.002 \cdot(t-20))} &
\end{array}\right\}(2)
$$

The four sets of parameters listed in Table I were obtained from real studies and used to simulate the myocardial tissue uptake using a two-compartment model:

$$
c(t)=f_{v} \cdot b(t)+\left(1-f_{v}\right) \cdot\left(k_{21} \cdot e^{-k_{12} \cdot t} \otimes b(t)\right)
$$

Here $c(t)$ is the measured uptake, $f_{v}$ is the vascular fraction of blood present in tissue, $k_{21}$ is the wash-in parameter and $k_{12}$ is the wash-out parameter.

Noise-free simulations were performed for every parameter set in Table I. For each set, $f_{v}$ was changed from 0 to 0.9 in steps of 0.1 and the time-activity curves were simulated using a two-compartment model. For each set, 50 identical curves representing myocardial uptake for each step of $f_{v}$ along with 200 identical curves representing the blood input function were obtained. Hence, a total of 700 curves were segmented with cluster analysis. A total of five clusters were obtained for each set of 700 curves from which the blood input function was selected as the cluster encompassing the blood region while the remaining clusters were fit to the two-compartment model. The clusters obtained were then passed on to the fuzzy k-means procedure to obtain a graded membership with each pixel. The pixels in the blood cluster were labelled as blood if they had a probability over a certain threshold; if not, they were assigned to the cluster with the next highest membership. An average of the probabilities of the most likely blood pixels selected manually was used as the threshold for the blood input finetuning procedure. This helped to remove non-blood pixels labelled as blood. The bias in the fits for both methods was calculated as the difference between the estimate and the truth divided by the truth.

The above simulations were also repeated after adding zero-mean Gaussian noise with intensity dependent variance to all the time-activity curves and computing the clusters with and without the use of blood input fine-tuning. The bias in the parameter estimates was also computed similar to that in the noise-free case. For all the sets, the mean and the standard deviation in the bias in the rate estimates for all the cluster fits (number of trials $=16$ ) was also computed.

\section{Canine and patient studies}

Eight studies (four teboroxime and four ${ }^{201} \mathrm{Tl}$ ) were performed in three canines. Among these, four studies (two teboroxime and two ${ }^{201} \mathrm{Tl}$ ) were performed at rest and four (two teboroxime and two ${ }^{201} \mathrm{Tl}$ ) with the adenosine stress protocol. In addition, three studies (one teboroxime rest, one teboroxime stress and one ${ }^{201} \mathrm{Tl}$ stress) were performed in two patients with dynamic cardiac SPECT. 
In two canine studies, an occlusion was placed on the left anterior descending artery while the third canine study was performed under normal physiological conditions. Radioactive microspheres (Sn-113, Ru-103) were used to obtain blood flows used as a gold standard. The tracer was injected over 5-10 sec (about $3 \mathrm{mCi}$ for ${ }^{201} \mathrm{Tl}$ and about 30 $\mathrm{mCi}$ for teboroxime) intravenously and the scan began $10 \mathrm{sec}$ before the tracer injection. In the case of stress studies, adenosine was infused over six minutes starting three minutes prior to the start of imaging. Complete sets of projection data (64 bins $\times 64$ slices $\times 120$ angles) were obtained with a dynamic sequence. For the teboroxime study, 180 frames of projection data were obtained with an acquisition time of 5.6 sec each. The ${ }^{201} \mathrm{Tl}$ study was performed in two stages with the first stage consisting of 60 frames each with an acquisition time of $10 \mathrm{sec}$ and the second stage with 30 frames each with an acquisition interval of $60 \mathrm{sec}$. The projection data for each study were reconstructed using the Ordered Subsets Expectation Maximization (OS-EM) algorithm with compensation for attenuation and detector response and reoriented into short-axis slices.

The dynamic cardiac SPECT protocol for patient studies was similar to the canine studies. The dynamic ${ }^{201} \mathrm{Tl}$ data were obtained in two stages with the first stage consisting of 60 frames of $10 \mathrm{sec}$ each and the second stage consisting of 60 frames of $60 \mathrm{sec}$ each. The dynamic teboroxime data on the other hand consisted of 90 frames with an acquisition time of $10 \mathrm{sec}$ each. All the dynamic frames were reconstructed and reoriented into short-axis slices.

The summed static data for the canine as well as patient studies were obtained by skipping the first $600 \mathrm{sec}$ (for ${ }^{201} \mathrm{Tl}$ ) or $100 \mathrm{sec}$ (for teboroxime) and summing the next $20 \mathrm{~min}$ (for ${ }^{201} \mathrm{Tl}$ ) or $10 \mathrm{~min}$ (for teboroxime) dynamic data. The summed static data were reconstructed and reoriented into short-axis slices and used with the semi-automatic method to obtain the regions of interest [7]. The semi-automatic method required the summed static short-axis slice data as input and found regions of interest based on a histogram specification technique. In this method, it is assumed that the short-axis slices are fairly circular and the regions of interest are found by selecting radii that have a specified Gaussian distribution. Moreover, the regions of interest on each slice are selected independently. More details regarding this method can be found in [7].

The short-axis dynamic frames were used for cluster analysis. The number of clusters chosen for each study was based on the noise present in the time-activity curve data. For example, in the case of teboroxime studies with relatively little noise, more clusters were chosen while for the ${ }^{201} \mathrm{Tl}$ studies, fewer clusters were selected. Based on this, 30 clusters were used for segmenting the 4D short-axis dynamic teboroxime canine data while 25 clusters were chosen for dynamic ${ }^{201} \mathrm{Tl}$ canine studies. The patient data for both dynamic teboroxime as well as dynamic ${ }^{201} \mathrm{Tl}$ were equally noisy and hence only 25 clusters were chosen. The threshold for the blood input fine-tuning process was selected by averaging the probabilities of the most likely blood pixels chosen manually. A two-compartment model was used to fit all the time activity curves using the blood input function obtained. The blood regions and regions that were not able to be fit, were set to zero by placing constraints on the kinetic rate parameters. This was done by removing clusters that had $f_{v}$ greater than 0.7 and also removing regions with negative or insensitive $k_{21}$ or $k_{12}$. The spatial locations of the remaining clusters were assigned the rate parameter estimates and hence parametric images were obtained.

In order to compare the rate parameter estimates obtained by cluster analysis and with the semi-automatic method, the regions obtained by semi-automatic analysis were used to obtain average kinetic rate parameter estimates from the corresponding regions in the clustered parametric images. The wash-in rate parameters were used to reflect flow. The flow data from the microspheres were obtained by well counting the tissue samples and visually registering the photographs of the myocardial slices with the summed static short-axis data for use as a gold standard [4]. The patient data were analyzed by comparing the wash-in rate parameters from the cluster analysis and the semi-automatic method to the summed intensities obtained from the summed static data.

The comparison of visual contrast of the flow data for all the methods was obtained from polar plots of the flow parameter. These polar plots were obtained by plotting either summed intensity or wash-in rate parameter obtained from myocardial regions of the short-axis data for all the slices from apex to the base, the apex being at the center. Five slices $(1 \mathrm{~cm}$ thick) were used for each study. In the case of microsphere flows and wash-in data from semi-automatic and clustering method, 4 regions per slice were used while for the summed static data, 80 regions per slice were used to create the polar map display.

\section{RESULTS}

\section{A. Simulations}

The true blood input function used for the simulations as well as those obtained using only standard k-means cluster analysis and the blood input fine-tuning procedure are shown in Fig. 2. It can be seen that the blood input function obtained with the fine-tuning procedure is closer to the true blood input function.

The mean bias in the kinetic rate parameter estimates using cluster analysis with and without fine-tuning for noise-free and noisy data are shown in Fig. 3. It can be seen that the bias in the case of using clustered blood is larger than that obtained using the blood input fine-tuning procedure. It can also be observed that the bias is even higher in the case of noisy data. The larger bias particularly in the wash-in rate parameters is likely due to the use of a contaminated input function in the case of standard k-means cluster analysis. 


\section{B. Canine and Patient studies}

The correlation coefficients between the microspherederived flow data and the wash-in parameters from clustering and from the semi-automatic method for all the canine studies are listed in Table II. It can be seen that the wash-in parametric data from the use of clustering gave better correlation with microspheres than did the semi-automatic technique. It was also observed that the use of the semiautomatic technique resulted in the failure of fitting routine to converge for some of the regions with dynamic ${ }^{201} \mathrm{Tl}$ data (hence the use of fewer regions for some studies). In particular, it can be observed that wash-in parameters with clustering for dynamic ${ }^{201} \mathrm{Tl}$ studies gave better correlation with the microsphere data than the semi-automatic method.

The scatter plots for the pooled canine data (no scaling performed) showing the relationship between the wash-in estimates and the microsphere flows are shown in Fig. 4. The wash-in estimates from using clustering were underestimated compared to using the semi-automatic method in the case of teboroxime studies, as evident from smaller slope in Fig. 4(b) as compared to that in Fig. 4(a). It can be also observed that the underestimation of flows in Fig. 4(b) occurs for flows greater than about $3 \mathrm{ml} / \mathrm{min} / \mathrm{g}$. On the other hand, it can be seen that the wash-in estimates from clustering gave better correlation with the microsphere flows compared to those from the semi-automatic method in the case of dynamic ${ }^{201} \mathrm{Tl}$ data (Fig. 4(c) and 4(d)).

The polar plots of the summed data, the wash-in parametric data with semi-automatic and the clustering method and the flow data from microspheres are shown in Fig. 5. The clustering method gave higher visual defect contrast compared to the semi-automatic method and the summed data. The summed data from the teboroxime studies gave lower defect contrast compared to the parametric plots. In the case of ${ }^{201} \mathrm{Tl}$ studies, the semi-automatic method overestimated the defect size while summed and wash-in parametric plots from clustering gave defect sizes comparable to the microsphere data with the wash-ins from clustering giving higher visual defect contrast than the summed data.

In the two patient studies performed, it was found that the semi-automatic method often failed to converge. On the other hand, the clustering method gave reasonable fits comparable to the summed static data in each case. Fig. 6 shows the polar plot of the summed and the wash-in parametric data from the use of clustering algorithm. It can be seen that the parametric data corresponds well with the summed data. In the teboroxime patient study, an X-ray angiography study suggested that the patient suffered from three-vessel disease. This was further validated by the low values of wash-in parameters obtained from fitting the time-activity curves of the ROIs. The summed and the parametric polar plots for the teboroxime study show a defect in the lateral wall indicating a possibility of a perfusion defect. The ${ }^{201} \mathrm{Tl}$ patient study was a normal study, which can be seen from the uniform parametric distribution in the polar plots. The wash-in polar plot gave uniform values for all regions and hence appears over-exposed.

\section{Discussion}

In the past we have used a semi-automatic technique to define regions of interest in dynamic SPECT images and obtain the time-activity curves. Several others perform the region of interest selection manually. Both of these methods are time-consuming and are based on selecting regions independently slice-by-slice based on the summed intensity distribution. In contrast, the clustering algorithm described here is based on partitioning the 4D data into groups of similar time-activity curves. This helps to select regions in three-dimensions and obtain less noisy curves resulting in better fits with the compartment model.

Other investigators have used different types of clustering algorithms for segmenting time-activity curve data with different imaging modalities. Wong et. al [8] used a weighted k-means clustering approach to segment dynamic FDG PET data for tumor analysis with simulations and real datasets. Their PET results indicated that the parameters obtained with cluster analysis were similar to those obtained with manual selection. Standard k-means clustering (unweighted) has been used by our group in dynamic contrast MRI studies to segment cardiac data and obtain a blood input function [10]. Kimura et. al [9] aimed at segmenting curves from dynamic brain FDG PET studies based on the curve shapes that were obtained by first normalizing the tissue uptake curves by the integral of the activity over the entire scan time. They then performed principal component analysis on the normalized curves to obtain principal components that were in turn used to segment the curves.

The simulation results indicated that the use of blood input fine-tuning following standard $\mathrm{k}$-means clustering helps to obtain a more accurate blood input function resulting in low parameter bias. The bias in the parameter estimates from the simulation data is partly due to fitting curves with different $f_{v}$ that were averaged during clustering, while the true estimates were obtained with curves where $f_{v}$ was set to zero. More work is required to validate the effects of using blood input fine-tuning on the bias in the parameter estimates.

The values of the wash-in rate parameters with clustering were lower than the corresponding semi-automatic method fits in the case of dynamic teboroxime data for flows greater than $3 \mathrm{ml} / \mathrm{min} / \mathrm{g}$ indicating a possibility of bias in the fits. This may be due to not using enough clusters. Further, the presence of background curves results in fewer clusters being selected from the myocardial region. Wong et. al [8] used two information-theoretic criteria as well as mean square error to determine the number of clusters to use. In the future, we propose to improve the algorithm by reducing the dependency on the number of clusters. Also, a hybrid method will be developed using information from individual pixel time activity curves along with our current clustering approach to obtain more accurate parametric maps. 
In the canine studies, it was observed that the cluster algorithm gave wash-in rate parameters that gave a good correlation with the microsphere derived flows. The use of clustering was also advantageous in the case of patient studies, where the semi-automatic method failed to converge due to the high statistical noise.

\section{CONCLUSION}

In summary, we developed an automated method of segmentation and parametric image formation for dynamic cardiac SPECT data. The method gives improved parameter estimates with noisy dynamic cardiac SPECT data. The use of this method offers potential for clinical applicability of dynamic cardiac SPECT imaging.

\section{ACKNOWLEDGMENT}

The authors would like to thank S. McJames and P. E. Christian for assistance in obtaining the canine data and $\mathrm{H}$. Elsamaloty, MD, for assistance in obtaining the patient data.

\section{REFERENCES}

[1] H. Iida and S. Eberl, "Quantitative assessment of regional myocardial blood flow with thallium-201 SPECT, " J. Nuc. Card., vol. 5, pp. 313$331,1998$.

[2] D. J. Kadrmas, E. V. R. DiBella, H. S. Khare, P. E. Christian and G. T. Gullberg, "Static versus dynamic teboroxime myocardial perfusion SPECT in canines, "IEEE Trans. Nucl. Sci., vol. 47, no. 3, pp. 1112$1117,2000$.

[3] E. V. R. DiBella, S. G. Ross, D. J. Kadrmas, H. S. Khare, P. E. Christian, S. McJames and G. T. Gullberg, "Compartmental modeling of technetium-99m-labeled teboroxime with dynamic SPECT: comparison with static Tl-201 in a canine model, " Invest. Rad., vol. 36, no. 3, pp. 178-185, 2000.

[4] H. S. Khare, E. V. R. DiBella, D. J. Kadrmas, P. E. Christian and G. T. Gullberg, "Comparison of static and dynamic cardiac perfusion thallium-201 SPECT, " IEEE Trans. Nucl. Sci., vol. 48, no. 3, pp. 774779, 2001.

[5] Y. Choi, R. A. Hawkins, S. Huang, et al. "Parametric images of glucose generated from dynamic cardiac PET and 2-[18F]fluoro-2-deoxy-dglucose studies, " J. Nucl. Med., vol. 32, pp. 733-738, 1991.

[6] Sitek A, Gullberg GT, Huesman RH, "Improved lesion contrast and reduced liver interference using parametric images, " J Nucl. Med. vol. 43, 144P, 2002.

[7] E. V. R. DiBella, G. T. Gullberg, A. B. Barclay and R. L. Eisner, "Automated region selection for analysis of dynamic cardiac SPECT data," IEEE Trans. Nucl. Sci, vol. 44, pp. 1355-1361, 1997.

[8] K. P Wong, D. Feng, S. R. Meikle, M. J. Fulham, "Segmentation of dynamic PET images using cluster analysis, "IEEE Trans. Nucl. Sci, vol. 49, no. 1, pp. 200-207, 2002.

[9] Y. Kimura, M. Senda and N. M. Alpert, "Fast formation of statistically reliable FDG parametric images based on clustering and principal components, " Phys. Med. Biol, vol. 47, pp. 455-468, 2002.

[10] E. V. R. DiBella and A. Sitek, "Time curve analysis techniques for dynamic contrast MRI studies, " Inf. Proc. in Med. Img., 17th Int. Conf. Proc, Springer, LNCS vol. 2082, 2001, pp. 211-217.

[11] R. O. Duda, P. E. Hart and D. G. Stork, Pattern Classification, 2nd ed., New York, Wiley, 2001. 


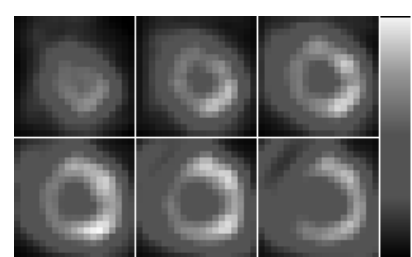

(a)

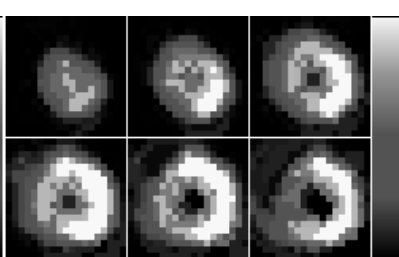

(b)

Fig. 1. Short-axis images from a teboroxime canine study. (a) is the summed short-axis image and (b) is the corresponding wash-in parametric image obtained using clustering (number of clusters $=30$ ).

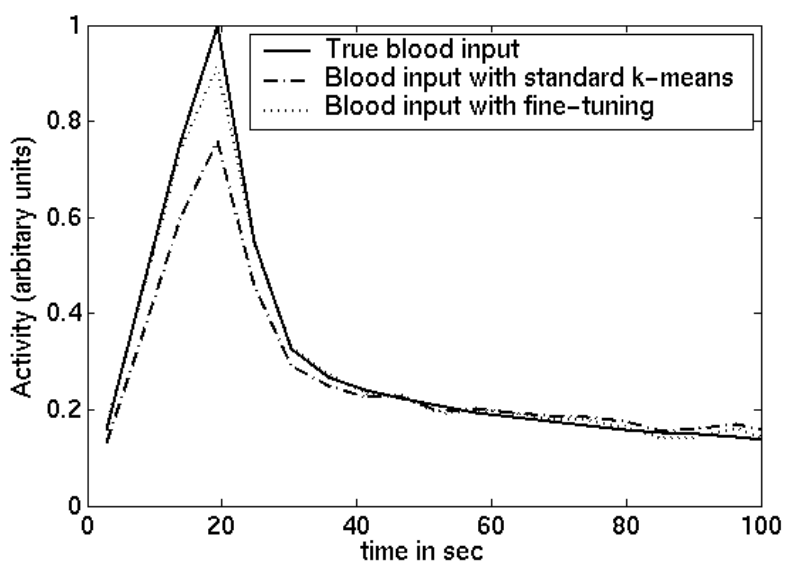

Fig. 2. Blood input function from the simulated data comparing the truth with that obtained with and without fine-tuning. The clustered blood input functions were obtained from the noisy simulations.

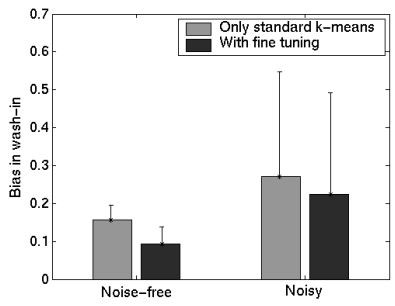

(a)

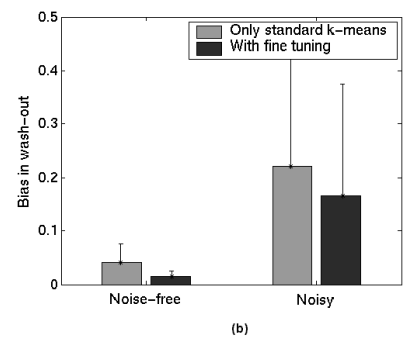

Fig. 3. Mean bias in the rate parameters from simulated data. Cluster analysis with blood input fine-tuning gives estimates with lower bias compared to using only standard k-means cluster analysis. 


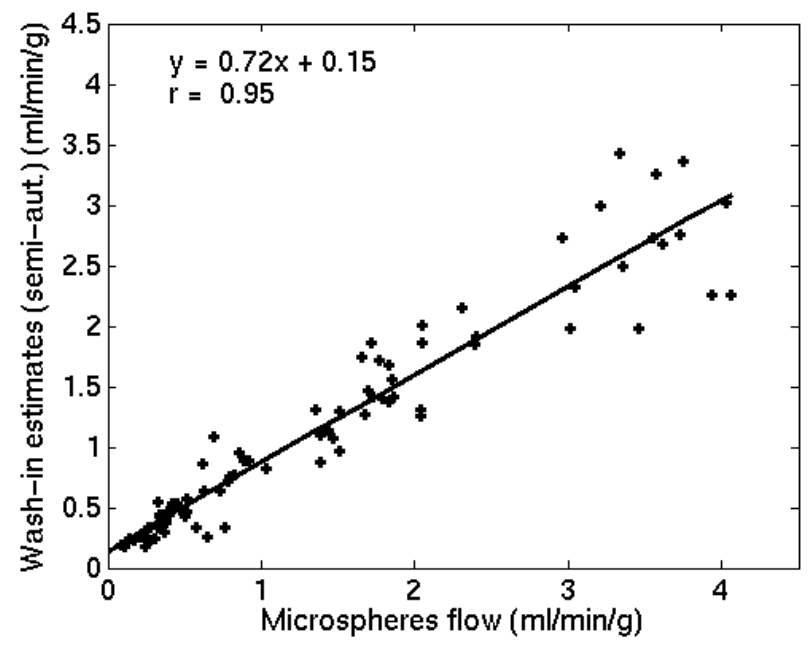

(a)

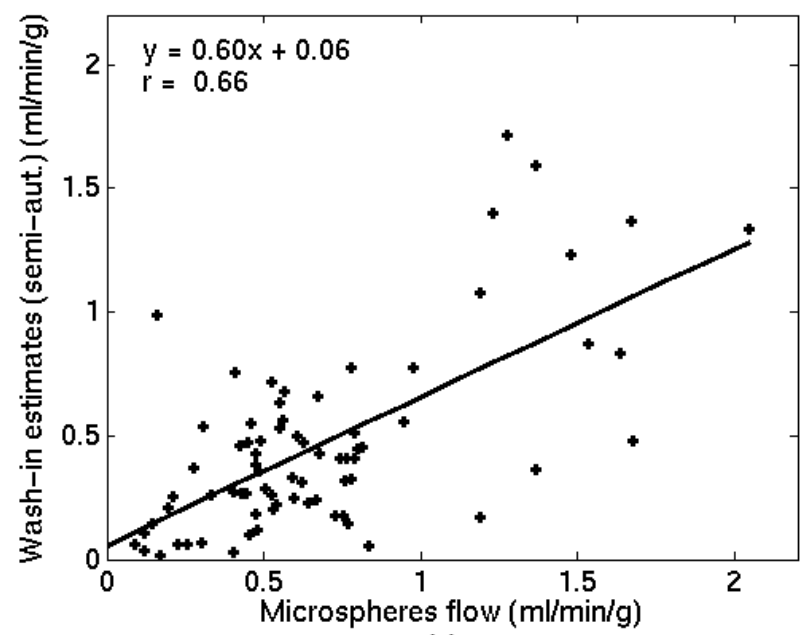

(c)

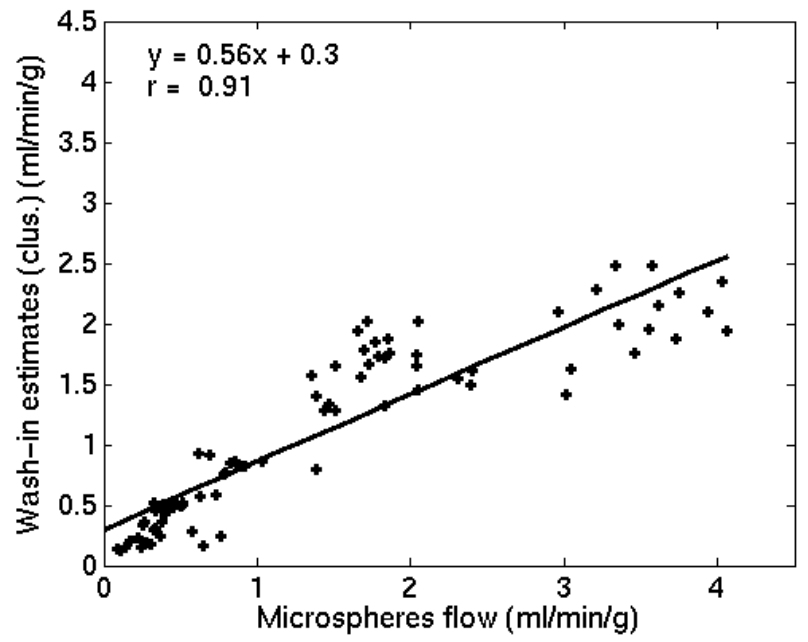

(b)

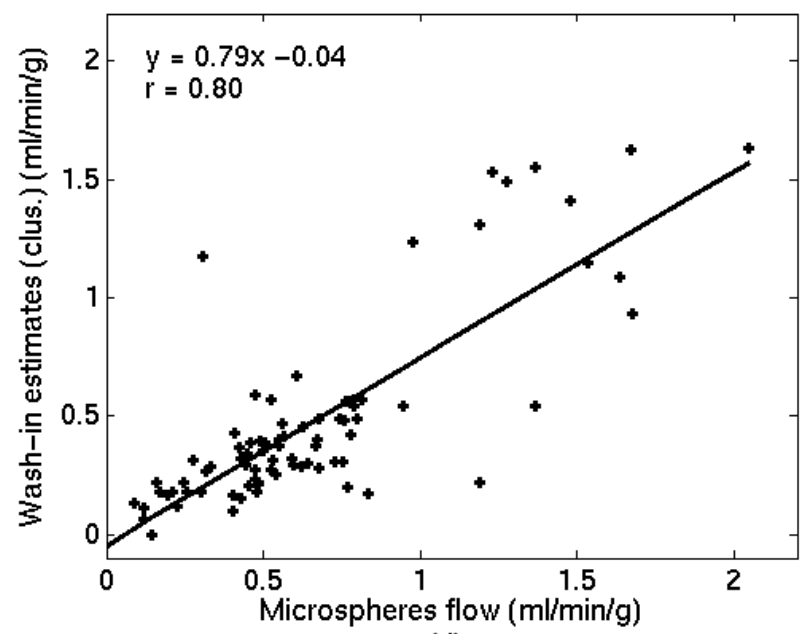

(d)

Fig. 4. Scatter plots for pooled canine data. (a) and (b) are plots of the wash-in parameter obtained using semi-automatic method and clustering respectively versus the microsphere flows for dynamic teboroxime data while (c) and (d) are plots of the wash-in parameter obtained using semi-automatic and clustering algorithm respectively versus the microsphere flows for dynamic ${ }^{201} \mathrm{Tl}$ data. 


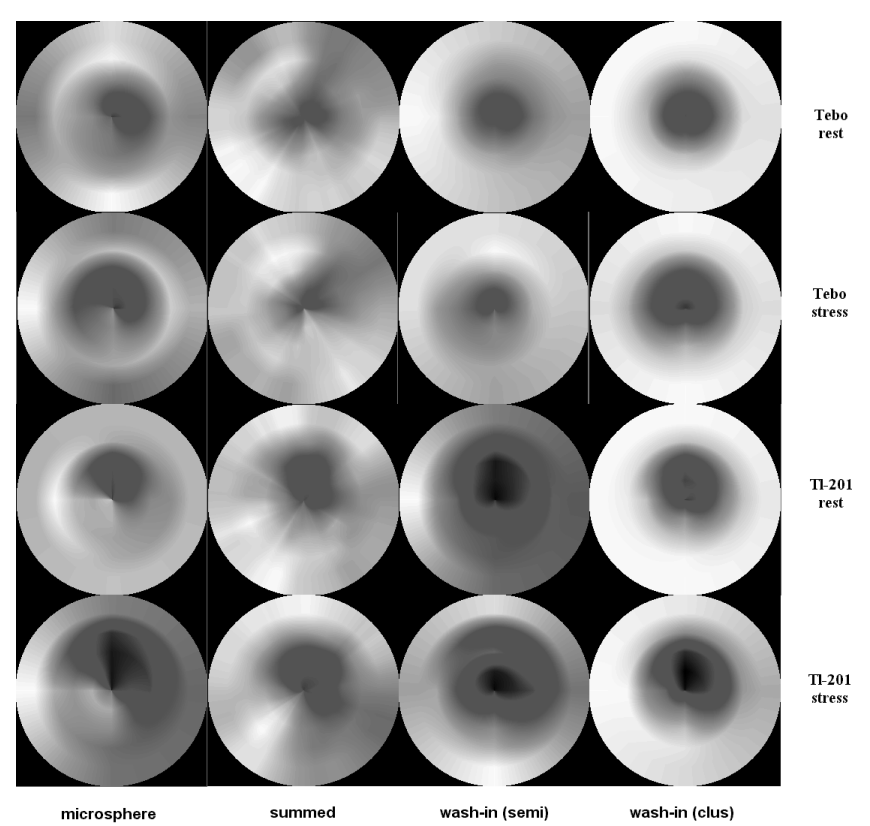

Fig. 5. Polar plots of the representative flows with different methods for two canine studies with rest and stress dynamic teboroxime and dynamic ${ }^{201} \mathrm{Tl}$ protocols. clus represents wash-in data from use of clustering and semi is the wash-in data obtained using the semi-automatic method.

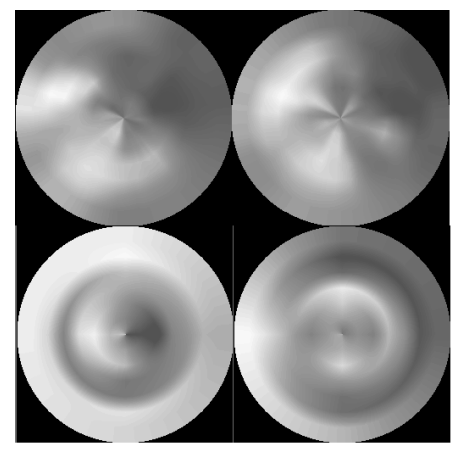

Tebo rest

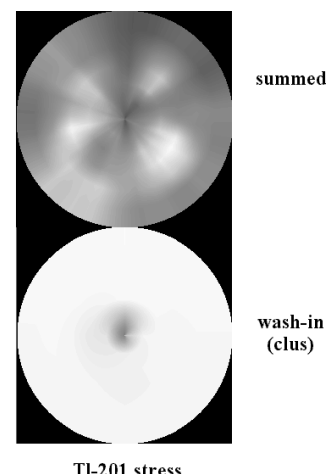

Tl-201 stress

Fig. 6. Polar plots of the flow representative data with summed and cluster analysis for patient studies. The teboroxime rest and stress studies were performed in one patient and the ${ }^{201} \mathrm{Tl}$ stress study was performed in another patient. 
Table I: Simulation Parameters

\begin{tabular}{|c|c|c|}
\hline Set & wash-in $\left(h_{21}\right) \min ^{-1}$ & wash-out $\left(h_{12}\right) \min ^{-1}$ \\
\hline 1 & 2.10 & 1.10 \\
\hline 2 & 0.72 & 0.72 \\
\hline 3 & 1.20 & 0.75 \\
\hline 4 & 0.52 & 0.47 \\
\hline
\end{tabular}

Table II: Correlation coefficients for canine studies

\begin{tabular}{|c|c|c|}
\hline Study & $\begin{array}{c}\text { wash-in } n_{\text {semi }} \text { vs. } \\
\text { microspheres }\end{array}$ & $\begin{array}{c}\text { wash-in } n_{\text {clx }} \text { vs. } \\
\text { microspheres }\end{array}$ \\
\hline Tebo rest 1 & $0.81(\mathrm{n}=20)$ & $0.81(\mathrm{n}=20)$ \\
\hline Tebo stress 1 & $0.72(\mathrm{n}=20)$ & $0.73(\mathrm{n}=20)$ \\
\hline Tebo rest 2 & $0.55(\mathrm{n}=20)$ & $0.67(\mathrm{n}=20)$ \\
\hline Tebo stress 2 & $0.61(\mathrm{n}=20)$ & $0.73(\mathrm{n}=20)$ \\
\hline${ }^{201}$ T1 rest 1 & $0.66(\mathrm{n}=15)$ & $0.69(\mathrm{n}=20)$ \\
\hline${ }^{201} \mathrm{~T} 1$ stress 1 & $0.66(\mathrm{n}=19)$ & $0.70(\mathrm{n}=20)$ \\
\hline${ }^{201}$ T1 rest 3 & $0.68(\mathrm{n}=20)$ & $0.72(\mathrm{n}=20)$ \\
\hline${ }^{201} \mathrm{~T} 1$ stress 3 & $0.58(\mathrm{n}=20)$ & $0.73(\mathrm{n}=20)$ \\
\hline
\end{tabular}

